\title{
Trends und Entwicklungen in der Hochspannungstechnik
}

\author{
H. M. Muhr OVE, CIGRE, IEC, IEE, DKE
}

Online publiziert: August 23, 2012

(C) Springer-Verlag 2012

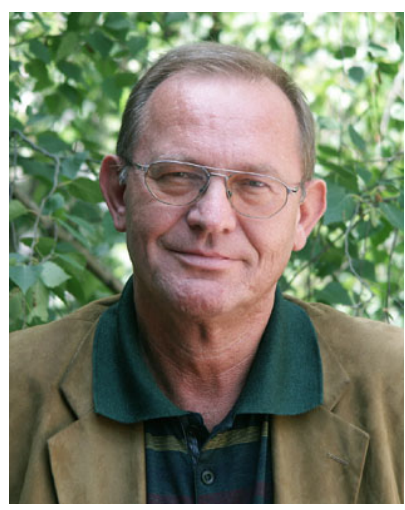

O. Univ.-Prof. Dipl.-Ing. Dr. Dr. h.c. Hans Michael Muhr
Liebe Leserinnen und Leser,

es ist mir eine große Ehre und Freude, dass es mir gestattet wurde, zu diesem Themenheft über die Hochspannungstechnik ein kurzes Vorwort sowie eine Einführung über die Hochspannungstechnik zu schreiben. Da ich seit mehr als 40 Jahren mit der Hochspannungstechnik verbunden bin, ist es für mich besonders erfreulich, dass sich die Redaktion von e\&i entschlossen hat, ein Themenheft in diesem Jahr der Hochspannungstechnik zu widmen. Um die fachliche sowie die internationale Breite der Hochspannungstechnik darzustellen, ist es uns gelungen, vier Beiträge von anerkannten Experten aus dem Ausland zu erhalten, während weitere Beiträge von den Damen und Herren des Instituts für Hochspannungstechnik und Systemmanagement der Technischen Universität Graz gestaltet wurden. Ich möchte daher an dieser Stelle allen Autorinnen und Autoren dafür herzlich danken. Durch ihre Mitwirkung hoffen wir, allen die Bedeutung und den Wert der Hochspannungstechnik darzustellen. Wir sind als praxisbezogene Ingenieure und Wissenschaftler unserer Aufgabe und Verantwortung wohl bewusst und versuchen daher, unsere Forschungen und Untersuchungen zum Wohle und zur Weiterentwicklung der Menschheit durchzuführen und für unsere Technische Universität Graz Wertvolles zu leisten.

Zentrale Aufgabe der Hochspannungstechnik ist der Umgang mit und die Beherrschung von hohen elektrischen Feldstärken und Spannungen, aber auch Strömen, wie etwa bei Blitzen. Hochspannung ist einerseits notwendig zur Übertragung großer elektrischer Leistungen, aber sie ist auch eine wichtige Größe für ein weiteres Spektrum technischer Anwendungen, wie etwa Röntgengeräte, Laser und Hochleistungslichtquellen, Senderöhren, Kopiergeräte, Elektrofilter und Beschichtungsanlagen. Aufgaben der Hochspannungstechnik bestehen auch bei der elektromagnetischen Verträglichkeit, der technologischen Forschung sowie bei der HochtemperaturSupraleitung. Im Spannungsfeld zwischen hohen elektrischen Feldstärken und der Vermeidung von Durchschlägen wird versucht, technische und wirtschaftliche Lösungen für die Systeme zu verwirklichen. Damit dies gelingen kann, sind für die Hochspannungstechnik neben der Elektrotechnik eine ganze Reihe von Wissenschaftszweigen erforderlich. In Abb. 1 soll dieser Zusammenhang aufgezeigt werden.

Beispiele für diese Wissensgebiete kommen durch die Isolierstof$\mathrm{fe}$, leitende Werkstoffe, Feldberechnung und Simulation, physikalisch und chemische Untersuchungen, mechanische und thermische

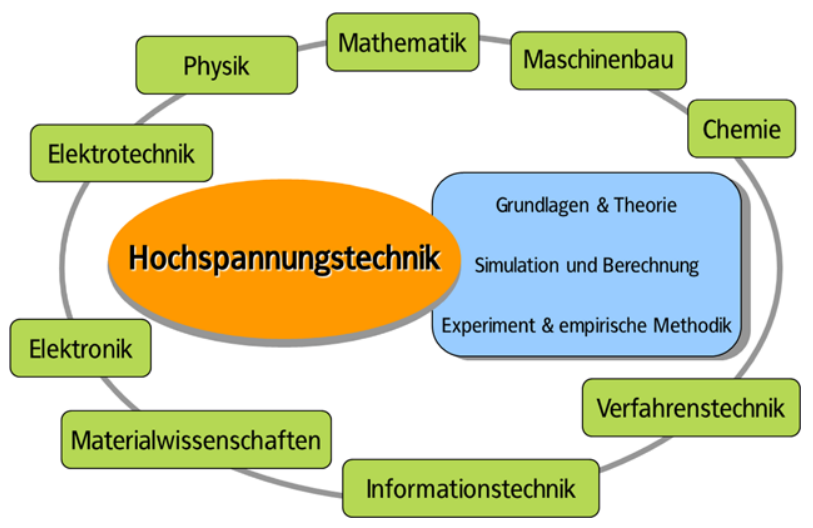

Abb. 1. Die Hochspannungstechnik im Zentrum zahlreicher Wissensgebiete

Auslegung, Prüf- und Messtechnik sowie Diagnostik, EMV, Signalverarbeitung, Informationstechnik, Leistungselektronik sowie Prozessautomatisierung. Die Personen, die sich daher mit Hochspannungstechnik beschäftigen, müssen vielseitig und praxisorientiert sein sowie einen gut fundierten Überblick über diese Wissensgebiete haben. Wobei in jüngster Zeit noch Fragen der Qualitätsoptimierung, des Prozessmanagements und der Risikobewertung eine wesentliche Rolle spielen. Wir am Institut versuchen diesen Aufgaben gerecht zu werden und haben daher auch unsere Lehr- und Forschungsbereiche diesen Wissensgebieten gewidmet. Dazu kommt noch die Versuchsanstalt für Hochspannungstechnik Graz GmbH, an der Prüfungen und Untersuchungen für die Industrie und Wirtschaft weltweit durchgeführt werden.

Daher soll dieses Themenheft, Ihnen, meine Damen und Herren, einen Einblick über die Hochspannungstechnik vermitteln und Ihnen zeigen, dass dieser Wissenschaftszweig zu einer Schlüsseltechnologie, man denke nur an den vieldiskutierten Bereich der Ultrahochspannungs-Übertragungstechnik, in unserer Gesellschaft geworden ist. Ich hoffe, Sie haben Freude an diesem Heft, und vielleicht erweckt die Hochspannungstechnik auch Ihr Interesse. Zudem erlaube ich mir zu erwähnen, dass von der Hochspannungstechnik in Graz die Sektion "Hochspannungstechnik" im OVE 1964 als Interessensgemeinschaft Hochspannungstechnik gegründet wurde, wobei 1964 auch die erste Fachaussprache abgehalten wurde. 1990 wurde dann diese Sektion in die "Österreichische Gesellschaft für Energietechnik (OGE)" umgewandelt.

Muhr, Hans Michael, 0. Univ.-Prof. Dipl.-Ing. Dr. Dr. h.c., Institut für Hochspannungstechnik und Systemmanagement, Technische Universität Graz, Graz, Österreich (E-Mail: muhr@tugraz.at) 